\title{
Unsupervised home monitoring of Parkinson's disease motor symptoms using body- worn accelerometers
}

James M. Fisher ${ }^{1}$, Nils Y. Hammerla ${ }^{2}$, Thomas Ploetz ${ }^{3}$, Peter Andras ${ }^{4}$, Lynn Rochester ${ }^{5}$, Richard W. Walker ${ }^{6}$

\section{Introduction}

Current PD assessment methods have inherent limitations. There is need for an objective method to assist clinical decisions and to facilitate evaluation of treatments. Accelerometers, and analysis using artificial neural networks (ANN), have shown potential as a method of motor symptom evaluation. This work describes the development of a novel PD disease state detection system informed by algorithms based on data collected in an unsupervised, home environment. We evaluated whether this approach can reproduce patient-completed symptom diaries and clinical assessment of disease state.

\section{Methods}

34 participants with PD wore bilateral wrist-worn accelerometers for $4 \mathrm{~h}$ in a research facility (phase 1) and for 7 days at home whilst completing symptom diaries (phase 2). An ANN to predict disease state was developed based on home-derived accelerometer data. Using a leave-one-out approach, ANN performance was evaluated against patient-completed symptom diaries and against clinician rating of disease state.

\section{Results}

In the clinical setting, specificity for dyskinesia detection was extremely high (0.99); high specificity was also demonstrated for home-derived data (0.93), but with low sensitivity (0.38). In both settings, sensitivity for on/off detection was sub-optimal. ANN-derived values of the proportions of time in each disease state showed strong, significant correlations with patient-completed symptom diaries.

\footnotetext{
${ }^{1}$ Department of Medicine, Northumbria Healthcare NHS Foundation Trust, United Kingdom

2 School of Computing Science, Newcastle University, United Kingdom

${ }^{3}$ School of Computing Science, Newcastle University, United Kingdom

${ }^{4}$ School of Computing and Mathematics, Keele University, United Kingdom

${ }^{5}$ Institute for Ageing and Health, Newcastle University, United Kingdom

${ }^{6}$ Department of Medicine, Northumbria Healthcare NHS Foundation Trust, United Kingdom
} 


\section{Conclusion}

Accurate, real-time evaluation of symptoms in an unsupervised, home environment, with this sensor system, is not yet achievable. In terms of the amounts of time spent in each disease state, ANN-derived results were comparable to those of symptom diaries, suggesting this method may provide a valuable outcome measure for medication trials.

Keywords: Parkinson's disease, body-worn sensors, home-monitoring

\section{Introduction}

Parkinson's Disease (PD) is a progressive, neurodegenerative dis-order characterised by motor symptoms, including tremor, rigidity and bradykinesia. With prolonged levodopa therapy, dyskinesia and motor fluctuations may develop [1]. The fluctuations of motor symptoms in PD make quantification of symptoms challenging. Current gold-standard assessment methods include clinical rating scales, most notably the Unified Parkinson's Disease Rating Scale (MDS-UPDRS) [2]. Whilst the MDS-UPDRS has undergone rigorous clinimetric validation, it remains time consuming to complete, and cannot feasibly be incorporated into routine practice. Patient-completed symptom diaries are also commonly used to evaluate patients' motor symptoms, but patient concordance with home diaries can be poor and entries are often not made contemporaneously [3]. Diary validity may be further compromised by incorrect disease state recognition [4], or by failure to recognise dyskinesia [5]. Furthermore, cognitive impairment has high prevalence within PD [6] and thus recall problems may adversely affect diary completion. Consequently, there is great need for an objective method of motor symptom assessment in PD.

Quantitative assessment of PD symptoms using body-sensors is increasingly recognised [79], yet the majority of work in this field has, thus far, focussed on gait [10]. Home monitoring of motor symptoms, using body-worn sensors, may enable more informed treatment decisions to be made, and development of such measures has been identified as a key research area for the PD community [11]. Such technology may provide a novel outcome 
measure for evaluation of new treatments [12]. The ability of body-worn sensors to accurately detect bradykinesia [13,14], tremor [14-16] and dyskinesia [17-22] has previously been shown. There are however, a number of limitations to the research thus far. In some instances the equipment employed has been bulky, with the potential to impair human movement [18]. Much of the work has required participants to remain stationary throughout the monitoring period $[15,16,19]$, thus precluding use of the technology in an unsupervised home monitoring environment. Previously, study participants have been asked to perform a set of scripted activities of daily living (ADL), whilst being monitored in a laboratory setting $[14,17,20-23]$ - thus findings may not be directly applicable to unconstrained naturalistic settings like the home. More prolonged monitoring in a home environment has been undertaken $[13,22]$ - however both studies failed to employ an alternative assessment method (e.g. symptom diaries) against which sensor findings could be validated. Furthermore, the symptom-detection algorithms underpinning both studies were derived from laboratory-based assessments, where patients performed predefined tasks whilst being monitored - again limiting their applicability to unsupervised home monitoring.

Artificial Neural Networks (ANN) are a type of probabilistic, para-metric model that consist of a large number of simple computational units arranged in a directed, layered structure [24]. ANN are capable of performing multifactorial analyses and have shown promise as analytical methods within the field of human biology [25]. The use of ANN in PD symptom measurement has been explored by a number of groups, with moderate success demonstrated for detection of dyskinesia $[17,23]$ and on/off disease states [26], albeit with data collection in controlled, 'supervised' environments.

If body-worn sensors are to provide a viable home symptom monitoring system for PD patients, greater consideration must be given to the ecological validity of data informing the symptom detection algorithms. This work describes the development of a novel PD disease state detection system that is informed by symptom detection algorithms based on human movement data collected in an unsupervised, home monitoring environment. In this work we 
investigate whether wrist-worn accelerometers, and data analysis with ANN, can reproduce:

(a) Patient-completed symptom diaries during prolonged periods of unobserved home monitoring; (b) Clinician assessment of PD patients' disease state during periods of observation in a clinical environment.

\section{Methods}

\subsection{Ethical approval}

This study was given a favourable ethical opinion by County Durham and Tees Valley Research Ethics Committee.

\subsection{Subjects and recruitment}

34 participants were recruited. All provided informed written consent. Participants were recruited from patients under the care of the Northumbria Healthcare NHS Foundation Trust PD service. Patients fulfilling the following inclusion criteria were recruited: Aged >18 years; Diagnosis of idiopathic PD (United Kingdom Brain Bank Criteria); Hoehn and Yahr stages IIV; Not significantly cognitively impaired (Mini-mental State Examination of $>24$ ); and taking immediate-release levodopa medication. Patient recruitment in this study aimed to include a range of motor disability, including a proportion of people with dyskinesia and motor fluctuations.

\subsection{Body-worn sensor}

The sensors employed in this study were the Axivity AX-3 [27] - a waterproof, tri-axial accelerometer attached via an adjustable Velcro strap (total weight $35 \mathrm{~g}$ ). The sensor can continuously sense for 12 days without recharging. Participants wore a sensor on each wrist for both study phases. The acceptability of prolonged, continuous wearing of these sensors, has previously been demonstrated [28].

Phase 1: Participants attended Newcastle University's Clinical Ageing Research Unit (CARU) and wore the sensors continuously for four hours. Participants were instructed to 
hold all antiparkinsonian medication after $10 \mathrm{pm}$ on the evening preceding their attendance and thus attended in the 'off disease state. All assessments began at 0900, but due to variations in treatment regimens the period of time that participants were off medication for was variable. During phase 1 participants were free to move around the department and to undertake unscripted ADLs. During their attendance at the clinic all participants underwent a complete MDS-UPDRS assessment. At hourly intervals participants underwent a clinical examination using a selection of MDS-UPDRS part III items assessing upper limb bradykinesia, tremor and gait (items 4,6,10,11,15-17) and on/off status was determined by clinician assessment. This was video-recorded and latterly reviewed by another member of the research team (RW), who undertook the same (blinded) assessment of the patient based on the recording. The above sub-set of the MDS-UPDRS part III was selected since it was judged to provide sufficient clinical assessment to enable the video-reviewer to pass judgement on motoric status, without being unduly burdensome in terms of time - too much time spent performing scripted activities would potentially render the data collected less ecologically valid.

After the initial assessment was completed participants took their usual anti-parkinsonian medications, meaning all participants took their first medications of the day at approximately the same time. Participants also received a standardised orientation ahead of the home monitoring phase - this included a review of what constitutes each of the motor states employed in the diaries, instruction as how to complete symptom diaries (including practice at doing so) and an explanation of the capabilities of the sensor.

Phase 2: Participants wore the sensors continuously at home for one week. Participants were asked to go about their normal ADLs. Participants also completed symptom diaries; recording, for each hour, whether they were predominantly 'on', 'off' , dyskinetic or asleep. Participants were advised to remove the sensors if they became unduly burdensome. At the end of the seven-day monitoring period the sensors and diaries were returned to the research team. 


\subsection{ANN development}

The 'traditional' approach to ANN development $[17,23,26]$, has been to initially collect data in a controlled, supervised manner and to then to use these data to 'train' an ANN to detect and/or measure symptoms. These algorithms are then tested against either unsupervised home data or against 'supervised', controlled, laboratory-derived data. Since laboratoryderived data cannot be considered ecologically valid we employed a novel approach. Here the ANN is 'trained' using a compendium of input (accelerometer) data and its corresponding output data (diary-defined disease state) - critically data are collected in the participants' homes in an unsupervised fashion, and can thus be considered ecologically valid.

From each minute of accelerometer recordings, from both the left and the right wrist, we extracted a number of signal characteristics that summarised the recordings. These included: Fourier coefficients, to capture repetitive movements; empirical cumulative distribution function features, that capture the distribution of acceleration magnitude and jerk; and statistical features including means and standard deviations. In total, 91 characteristics were extracted from each minute - a more detailed description of this process, from a computing science perspective, has previously been published [29].

The ANN used in this work took the characteristics of 5 consecutive minutes as input. It contained two fully connected hidden layers with 2048 (logistic) units each. The top layer contained one unit for each target class: asleep, off, on, and dyskinesia. The parameters of the ANN were optimized (trained) based on a subset of the recording (the training-set) in a two-step process. First, an unsupervised learning algorithm was applied to initialise the weights of all connections in the ANN, as described previously [30]. Next, a supervised approach compared the output of the ANN with the annotations provided by the participants (diary), and adjusted the weights by propagating the derivative of the disagreement (error) backwards through the network. The motor state reported by participants for a given one hour diary interval, was taken to represent the motor state for all 5 min windows within that hour. The subsets used for training and evaluation of the ANN are constructed following a 
leave-one-day-out approach. A more detailed description of the training process applied in this work has previously been published [29].

The result of the training process was an ANN that, for an input of 5 consecutive minutes, predicted a probability distribution over the 4 classes of interest. The value for a specific class, e.g. dyskinesia, represents the confidence of the ANN that the input contains dyskinesia. A prediction is made by selecting the class with the highest confidence.

\subsection{ANN performance evaluation}

The performance of the ANN was evaluated using both supervised, laboratory-derived data and unsupervised, home-derived data. Critically, performance was evaluated using unseen data to ensure that results were not confounded by the ANN simply 'learning' data it had previously 'seen'. When evaluating ANN performance on home data, ANN predictions were compared against diary entries. To explore criterion-related validity, correlations between the amounts of time in a given disease state, as measured by ANN, home diaries and MDS-UPDRS part IV, were examined using Pearson's correlation coefficient. For laboratory-derived accelerometer data, data from ten minute periods before and after the formal assessments were taken and divided into one minute epochs. The accelerometer data obtained during the clinical assessments themselves were excluded, since activities performed as part of an abbreviated MDS-UPRDS are highly scripted and cannot be considered ecologically valid. When evaluating ANN performance of previously unseen laboratory-derived data, ANN predictions were compared to clinicians' assessment of disease status.

\section{Results}

\subsection{Participants}

The mean age of participants was 68.9 years $(S D=9.1)$. Mean disease duration was 9.8 years $(S D=5.6)$. Median Hoehn and Yahr Stage was II (range I-IV). The disease phenotype of participants was determined (as per Stebbins et al. [31]) - 12 tremor dominant, 19 postural instability/gait difficulty and 3 indeterminate. All patients were on anti-parkinsonian 
medication with a mean levodopa equivalent dose [32] of $918.1 \mathrm{mg} /$ day. 28 (82.4\%) were taking dopamine agonists, 6 (17.6\%) were taking monoamine oxidase B inhibitors, 12 (35.3\%) were taking catechol-o-methyl transferase inhibitors, 7 (20.6\%) were taking amantadine and 2 (5.9\%) were using continuous intra-jejunal infusion of levodopa-carbidopa intestinal gel. 14/34 participants (41.2\%) experienced dyskinesia and, based on symptom diaries, 31/34 experienced motor fluctuations. Cognitive assessment with MMSE revealed a mean score of $28.6(S D=1.5)$. Mean MDS-UPDRS scores by section were: I - 15.9 $(\mathrm{SD}=5.6) ;$ II - 20.1 (SD = 9.7); III - $32.9(\mathrm{SD}=14.4)$ and IV - $6.9(\mathrm{SD}=3.6)$.

\subsection{Diary data}

All 34 participants completed diaries during phase II; 32 did so for the entire seven days. If, for a given time period, no diary entry was made, or if two or more disease states were selected, these entries were deemed 'unclassifiable' . The mean percentage of classifiable responses across the study cohort was $82.5 \%(S D=26.2)$.

\subsection{ANN performance}

A total of $332,640 \mathrm{~min}(5544 \mathrm{~h})$ of accelerometer data were recorded from the participants at home with a frequency of $100 \mathrm{~Hz}$ (samples per second). 268,388 min (4473 h, 80.7\%) had corresponding diary entries. In the laboratory setting a total of $1195 \min (\sim 20 \mathrm{~h})$ were extracted surrounding the clinician's assessments. Participants' motor status was dual-rated by two clinicians - assessments where the two clinicians disagreed on disease status were discarded (inter-rater agreement seen in $>95 \%$ of assessments). Data were extracted surrounding the remaining 141 phase 1 clinical assessments. Fig. 1 (a) displays a normalised confusion matrix which shows agreement between diary-defined disease status (y-axis) and sensor-determined disease status (x-axis). Fig. 1(b) displays a confusion matrix that shows clinician-defined disease status (y-axis) and sensor-determined disease status (x-axis). 
Sensitivity and specificity data for each disease state, for both home and laboratory data, are presented in Table 1. Values for 'asleep' in the laboratory setting are not presented, since this 'disease state' was not encountered during laboratory data collection.

Correlations between measurements of the amounts of time produced by differing assessment methods, for a given disease state, were evaluated using Pearson's correlation coefficient. Results are displayed in Table 2 below, where consideration is given to home diaries vs. ANN, and MDS-UPDRS (Part IV) vs. home diaries. Correlation data are presented for 'excellent' diarists (where completion was >75\%; $n=25$ ) and 'good' diarists (where completion was $>50 \% ; n=29$ ).

For data collected during home monitoring periods, graphical representations of participants' actual disease states (determined from diaries) and predicted disease states (determined by ANN) were produced. Examples are shown in Fig. 2 that demonstrate, for four participants, how hourly data is charted to provide an overview of the disease states experienced during a period of three days. The daily average time spent in each disease state, as determined by the ANN, diaries and by the MDS-UPDRS part IV are also displayed.

\section{Discussion}

Previous attempts to translate algorithms based on laboratory-derived data to home monitoring of upper limb motor symptoms have had major limitations, since the lab-based data informing their development lacked ecological validity. The innovative approach employed in this work captured, through home monitoring, a very large quantity of real-life data to inform algorithm development. A further strength was the use of patient-completed symptom diaries, the current gold standard for home monitoring, as the anchor for algorithm development.

In the laboratory, specificity for dyskinesia detection was extremely high (0.99). High specificity was also demonstrated for home-derived data (0.93), but with low sensitivity (0.38). A possible contributory factor to this sub-optimal performance is that patients may 
have experienced dyskinesia in body segments other than the upper limbs, thus rendering the wrist-worn sensors less sensitive. Superior performance was evident in the laboratory environment since clinicians are more likely than patients to identify subtle dyskinesia [5]. Comparative work, using ANNs to assess dyskinesia, highlighted similar challenges with detection of mild dyskinesia, particularly in the presence of volitional movement $[17,23]$. Notably, these ANNs were developed using laboratory-derived data, and required six sensors - the acceptability of such systems to the wearer has not been demonstrated. In both home and laboratory settings, sensitivity for on/off detection was sub-optimal (on: $0.52,0.69$; off: $0.50,0.60$ ); whilst higher specificity values were demonstrated (on: 0.91, 0.82; off: $0.83,0.83)$. Previous work by Keijsers et al. [26] employed six body-worn sensors and developed an ANN to detect on/off states, reporting extremely high sensitivity and specificity $(0.99 \& 0.99)$. However, these results were based on training data alone - the ANN was not applied to unseen data, and thus these results simply represent over-training, effectively 'memorisation' of the data [25]. On/off detection is rendered extremely challenging due to the inherent subjectivity underlying interpretation of disease state descriptors - exactly what degree of motor symptoms each participant deems to be on/off is likely to differ between individuals. The challenges of identifying on/off states are compounded by the use of an hourly diary as the anchor against which the ANN was developed. For many of our participants, disease state was liable to fluctuation, and thus participants likely experienced more than one disease state in a given hour. Hourly intervals were employed in an attempt to minimise the burden of diary completion, due to concern about the potential negative effect burdensome diaries could have on both diary concordance and accuracy. We do however acknowledge that symptom diaries based on a 30 min interval are widely used in PD research [33] and may provide improved data for future work.

Whilst patient-completed symptom diaries are the current gold standard for home monitoring, they do have other inherent limitations. Whilst the high diary-completion rates seen in this work are reassuring, we acknowledge that the presence of a diary entry does 
not guarantee that the entry accurately represents a person's disease state at that time. Despite these limitations, diaries are however the most practicable current home assessment method. Future work should consider implementing a more rigorous diary orientation exercise during phase one: firstly, this may improve diary accuracy during home monitoring periods; secondly, it would enable agreement between clinician and participant to be evaluated, potentially providing external validation of diary content; and lastly, it would allow patient-defined disease state to be employed as the 'ground truth' for data collected in the clinical environment.

Consideration was also given to the amounts of time spent in each disease state as recorded by diaries, predicted by ANN and estimated by MDS-UPDRS part IV. Correlation data between assessment methods (Table 2) provides evidence for the criterion-related validity of the ANN assessment method, since strong correlations were seen between ANN predictions and actual diary values. This is an important finding, since the amounts of time in a given disease state may provide a valuable outcome measure for medication trials. The use of symptom diaries in clinical trials often necessitates exclusion of cognitively impaired patients, for whom diary completion may be challenging and potentially inaccurate, thus limiting the generalisability of findings. In our work, diary completion was worse in patients with lower MMSE scores (Mann Whitney $U$ test, $Z=-2.275, p=0.023$ ). Discrete body-worn sensors capable of recording disease status may therefore provide an attractive assessment method for cognitively impaired patients. We acknowledge that cognitively impaired patients were excluded from our work and that their sensor concordance cannot be assumed.

The ideal PD home monitoring system would enable real-time evaluation of symptoms to the extent where medication dosing and timing could be adjusted in light of the information obtained. Fig. 2 shows examples of real-time disease state estimations with diary entries superimposed for comparison. It was not possible to produce similarly accurate traces for all study participants due to disparity between diary entries and sensor predictions, highlighting that real-time evaluation with this sensor system, is not yet achievable. 
In contrast to previous work employing ANN, where a multitude of sensors were employed across all four limbs, the trunk and chest, our assessment method used just two wrist-worn sensors. Using just two wrist-worn sensors meant less data were available to describe human movement and furthermore, interpretation of data was rendered more challenging due to the greater amount of voluntary movement exhibited in the upper limbs. The benefit of this approach is patient acceptability and wearability in a home environment [28]. Irrespective of the analysis approach employed, there is a lack of consensus in the literature regarding the optimal number of sensors, and the optimal site for their placement, for the assessment of PD motor symptoms - addressing this lack of conclusive data has been highlighted as a key challenge facing the field [9]. Despite these challenges our work provides evidence of the criterion-related validity of this assessment system. Rigorous scientific evaluation of an outcome measurement instrument also requires consideration of reliability and responsiveness [34] - thus further work is needed to evaluate these properties.

Body-worn technology has huge potential to change how the motor symptoms of PD are measured. As technology advances, body-worn sensors may be capable of functioning as 'data processing' sensors, enabling automated, real-time evaluation of data and thus providing the wearer with rapid feedback [35]. Future possibilities include modification of a patient's medication regimen in response to real-time data analysis, or even integration of such systems with non-oral medication delivery systems [36]. However, if such technology is to be translated into a meaningful tool for research use and clinical practice, it is essential that the ecological validity of data underpinning the algorithms is considered.

\section{Financial disclosure/conflict of interest}

There are no conflicts of interest. LR is supported by the National Institute for Health Research (NIHR) Newcastle Biomedical Research Unit based at Newcastle upon Tyne Hospitals NHS Foundation Trust and Newcastle University. The research was also supported by NIHR Newcastle CRF Infrastructure funding. The views expressed are those of the authors and not necessarily those of the NHS, the NIHR or the Department of Health. 


\section{Funding}

This study was funded by Northumbria Healthcare NHS Foundation Trust.

\section{Acknowledgments}

The authors would like to thank the participants who willingly gave up their time to contribute to this work. The authors would also like to thank the Movement Disorders Service at Northumbria Healthcare NHS Foundation Trust for their help and support.

\section{References}

[1] O. Rascol, D.J. Brooks, A.D. Korczyn, P.P. De Deyn, C.E. Clarke, A.E. Lang, A five-year study of the incidence of dyskinesia in patients with early Parkin-son's disease who were treated with ropinirole or levodopa, N. Engl. J.

Med. 342 (20) (2000) 1484-1491.

[2] C.G. Goetz, B.C. Tilley, S.R. Shaftman, G.T. Stebbins, S. Fahn, P. Mar- tinez-Martin, W. Poewe, C. Sampaio, M.B. Stern, R. Dodel, B. Dubois, R. Hol-loway, J. Jankovic, J. Kulisevsky, A.E. Lang, A. Lees, S. Leurgans, P.A. Le- Witt, D. Nyenhuis, C.W. Olanow, O. Rascol, A. Schrag, J.A. Teresi, J.J. van Hilten, N. LaPelle, Movement disorder societysponsored revision of the unified Parkinson's disease rating scale (MDS-UPDRS): scale presentation and clini- metric testing results, Mov. Disord. 23 (15) (2008) 2129-2170.

[3] A.A. Stone, S. Shiffman, J.E. Schwartz, J.E. Broderick, M.R. Hufford, Patient noncompliance with paper diaries, Br. Med. J. 324 (2002) 1193-1194.

[4] C.G. Goetz, G.T. Stebbins, L.M. Blasucci, M.S. Grobman, Efficacy of a pa-tienttraining videotape on motor fluctuations for on-off diaries in Parkinson's disease, Mov. Disord. 12 (6) (1997) 1039-1041.

[5] C. Vitale, M.T. Pellecchia, D. Grossi, N. Fragassi, T. Cuomo, L. Di Miao, P. Barone, Unawareness of dyskinesias in Parkinson's and Huntington's diseases, Neurol. Sci. 22 (2001) 105-106.

[6] D. Weintraub, D.J. Burn, Parkinson's disease: the quintessential neuropyschi- atric disorder, Mov. Disord. 26 (6) (2011) 1022-1031.

[7] W. Maetzler, J. Domingos, K. Srulijes, J.J. Ferreira, B.R. Bloem, Quantitative wearable sensors for objective assessment of Parkinson's disease, Mov. Dis $\neg$ ord. 28 (12) (2013) 1628-1637.

[8] W. Maetzler, L. Rochester, Body-worn sensors-the brave new world of clinical measurement?, Mov. Disord. 30 (9) (2015) 1203-1205.

[9] C. Ossig, A. Antonini, C. Buhmann, J. Classen, I. Csoti, B. Falkenburger, M. Schwarz, J. Winkler, A. Storch, Wearable sensor-based objective assessment of motor symptoms in Parkinson's disease, J. Neural Transm. 123 (1) (2016) 57-64. 
[10] L. Rochester, K. Baker, A. Nieuwboer, D. Burn, Targeting dopa-sensitive and doparesistant gait dysfunction in Parkinson's disease: selective responses to internal and external cues, Mov. Disord. 26 (3) (2011) 430-435.

[11] K.H.O. Deane, H. Flaherty, D.J. Daley, R. Pascoe, B. Penhale, C.E. Clarke, C. Sackley, S. Storey, Priority setting partnership to identify the top 10 research priorities for the management of Parkinson's disease, BMJ Open 4 (12) (2014).

[12] J.R. Evans, R.A. Barker, Defining meaningful outcome measures in trials of dis-easemodifying therapies in Parkinson's disease, Expert Opin. Pharma- cother. 12 (8) (2011) 1249-1258.

[13] R.I. Griffiths, K. Kotschet, S. Arfon, Z.M. Xu, W. Johnson, J. Drago, A. Evans, P. Kempster, S. Raghav, M.K. Horne, Automated assessment of bradykinesia and dyskinesia in Parkinson's disease, J. Park. Dis. 2 (1) (2012) 47-55.

[14] A. Salarian, H. Russmann, C. Wider, P.R. Burkhard, F.J.G. Vingerhoets, K. Aminian, Quantification of tremor and bradykinesia in Parkinson's disease using a novel ambulatory monitoring system, IEEE Trans. Biomed. Eng. 54 (2) (2007) 313-322.

[15] S. Mellone, L. Palmerini, A. Cappello, L. Chiari, Hilbert-Huangbased tremor re-moval to assess postural properties from accelerometers, IEEE Trans. Biomed. Eng. 58(2011) 1752-1761.

[16] I. Schlesinger, O. Benyakov, I. Erikh, S. Suraiya, Y. Schiller, Parkinson's disease tremor is diminished with relaxation guided imagery, Mov. Disord. 24 (14) (2009) 2059-2062.

[17] N.L.W. Keijsers, M.W.I.M. Horstink, S.C.A.M. Gielen, Automatic assessment of levodopa-induced dyskinesias in daily life by neural networks, Mov. Disord. 18 (1) (2003) 70 80.

[18] A.J. Manson, P. Brown, J.D. O'Sullivan, P. Asselman, D. Buckwell, A.J. Lees, An ambulatory dyskinesia monitor, J. Neurol. Neurosurg. Psychiatry 68 (2) (2000) 196-201.

[19] T.O. Mera, M.A. Burack, J.P. Giuffrida, Objective motion sensor assessment highly correlated with scores of global levodopa-induced dyskinesia in Parkinson's disease, J. Park. Dis. 3 (3) (2013) 399-407.

[20] C.L. Pulliam, M.A. Burack, D.A. Heldman, J.P. Giuffrida, T.O. Mera, Motion sensor dyskinesia assessment during activities of daily living, J. Park. Dis. 4 (4) (2014) 609-615.

[21] G. Lopane, S. Mellone, L. Chiari, P. Cortelli, G. Calandra-Buonaura, M. Contin, Dyskinesia detection and monitoring by a single sensor in patients with Parkin-son's disease, Mov. Disord. 30 (9) (2015) 1267-1271.

[22] R. Ramsperger, S. Meckler, T. Heger, J. van Uem, S. Hucker, U. Braatz, H. Graessner, D. Berg, Y. Manoli, J.A. Serrano, J.J. Ferreira, M.A. Hobert, W. Maetzler, Continuous leg dyskinesia assessment in Parkinson's disease - clinical validity and ecological effect, Park. Relat. Disord. 26 (2016) 41-46. 
[23] M.G. Tsipouras, A.T. Tzallas, D.I. Fotiadis, S. Konitsiotis, On automated assessment of Levodopa-induced dyskinesia in Parkinson's disease, In: Conf Proc IEEE Eng Med Biol Soc, 2011 Annual International Conference of the IEEE, 2011, pp. 2679-2682.

[24] C. Bishop, Pattern Recognition and Machine Learning, Springer-Verlag, New York, 2006.

[25] J.E. Dayhoff, J.M. DeLeo, Artificial neural networks, Cancer 91 (2001) 1615-1635.

[26] N.L.W. Keijsers, M.W.I.M. Horstink, S.C.A.M. Gielen, Ambulatory motor as-sessment in Parkinson's disease, Mov. Disord. 21 (1) (2006) 34-44. Date of Publication: Jan 2006.2006.

[27] Axivity AX3 3 Axis Logging Accelerometer: Data Sheet, 2013, (accessed 29.07.16).

[28] J.M. Fisher, N.Y. Hammerla, L. Rochester, P. Andras, R.W. Walker,

Body-worn sensors in Parkinson's disease: evaluating their acceptability to patients, Telemed. J. E Health 22 (1) (2015) 63-69.

[29] N.Y. Hammerla, J. Fisher, P. Andras, L. Rochester, R. Walker, T. Ploetz, PD disease state assessment in naturalistic environments using deep learning, In: Proceedings of the Twenty-ninth AAAI Conference on Artificial Intelligence, Austin, Texas, 2015.

[30] G.E. Hinton, R.R. Salakhutdinov, Reducing the dimensionality of data with neural networks, Science 313 (5786) (2006) 504-507.

[31] G.T. Stebbins, C.G. Goetz, D.J. Burn, J. Jankovic, T.K. Khoo, B.C. Tilley, How to identify tremor dominant and postural instability/gait difficulty groups with the movement disorder society unified Parkinson's disease rating scale: comparison with the unified Parkinson's disease rating scale, Mov. Disord. 28 (5) (2013) 668-670.

[32] C.L. Tomlinson, R. Stowe, S. Patel, C. Rick, R. Gray, C.E. Clarke, Systematic review of levodopa dose equivalency reporting in Parkinson's disease, Mov. Disord. 25 (15) (2010) 2649-2653.

[33] R.A. Hauser, F. Deckers, P. Lehert, Parkinson's disease home diary: further validation and implications for clinical trials, Mov. Disord. 19 (12) (2004) 1409-1413.

[34] J.C. Hobart, D.L. Lamping, A.J. Thompson, Evaluating neurological outcome measures: the bare essentials, J. Neurol. Neurosurg. Psychiatry 60 (1996) 127-130.

[35] C. Ni Scanaill, S. Carew, P. Barralon, N. Noury, D. Lyons, G.M. Lyons, A re $\neg$ view of approaches to mobility telemonitoring of the elderly in their living environment, Ann. Biomed. Eng. 34 (4) (2006) 547-563. 
[36] A. Rodriguez-Molinero, D.A. Perez-Martmez, A. Catala, J. Cabestany, A. Yuste, Treatment of Parkinson's disease could be regulated by movement sensors: subcutaneous infusion of varying apomorphine doses according to the intensity of motor activity, Med. Hypotheses 72 (4) (2009) 430-433.

\section{Figures \& Tables}

\section{Figure 1}

Confusion matrices of: (a) predicted disease state against actual (diary) disease state for home data; (b) predicted disease state against actual (clinician-rated) disease state for laboratory data [numerical values represent the number of five minute epochs for HOME data and the number of one minute epochs for LAB data].
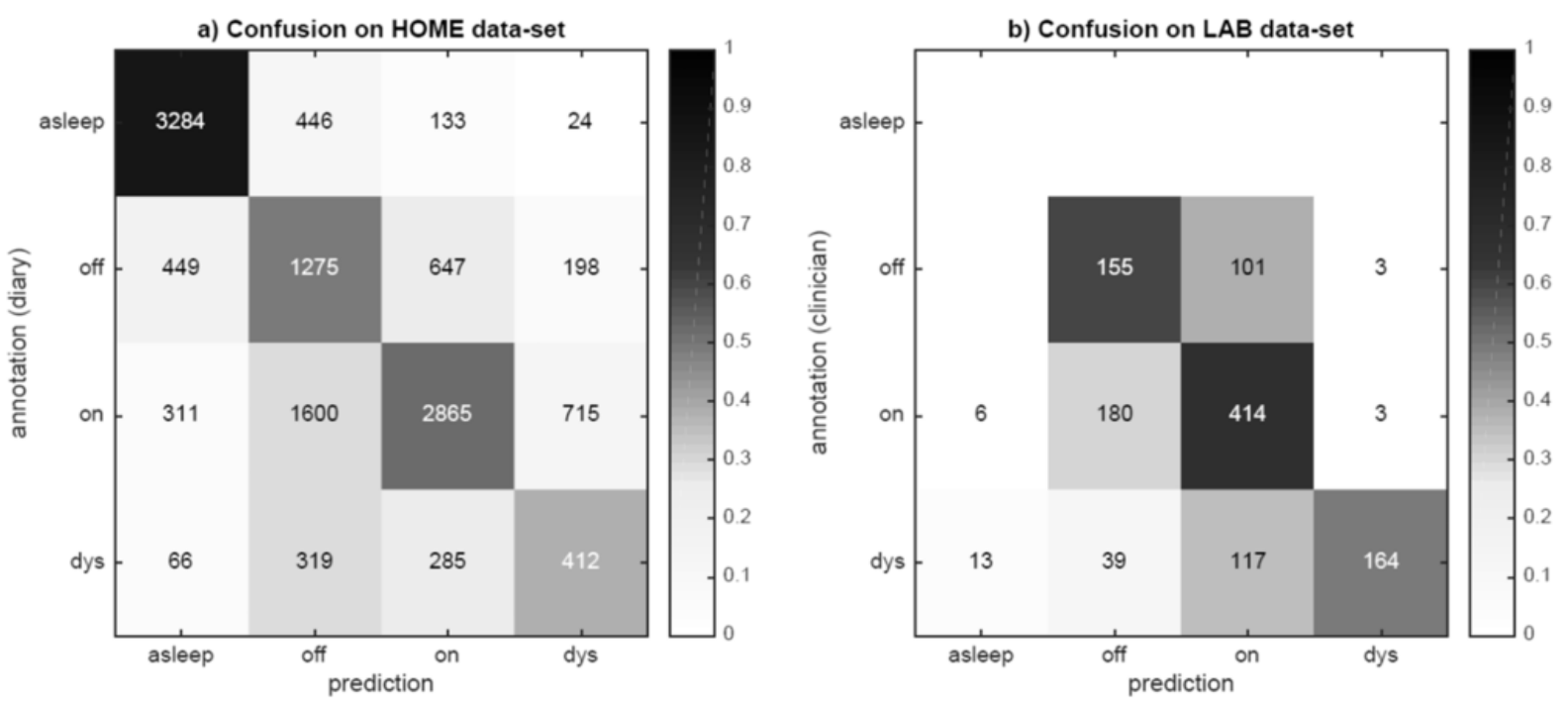

Table 1

Sensitivity and Specificity Data for each disease state, for both home and laboratory data.

\begin{tabular}{lllll}
\hline & Home & & Laboratory & \\
\hline & Sensitivity & Specificity & Sensitivity & $\begin{array}{l}\text { Specificit } \\
\text { y }\end{array}$ \\
\hline Asleep & 0.85 & 0.94 & $\mathrm{n} / \mathrm{a}$ & $\mathrm{n} / \mathrm{a}$ \\
Off & 0.50 & 0.83 & 0.60 & 0.83 \\
On & 0.52 & 0.91 & 0.69 & 0.82 \\
Dyskinesia & 0.38 & 0.93 & 0.49 & 0.99 \\
Mean & 0.56 & 0.90 & 0.59 & 0.88 \\
& & & & \\
\hline
\end{tabular}




\section{Table 2}

Correlation between the amounts of time in a given disease state (MDS-UPDRS part IV vs. patient-completed diary and diary vs. ANN) for both 'excellent' and 'good' diarists.

\begin{tabular}{|c|c|c|c|c|c|}
\hline & \multicolumn{2}{|c|}{ Diary vs. ANN } & \multicolumn{2}{|c|}{ UPDRS vs. Diary } \\
\hline & & $r$ & $P$ & $\mathbf{r}$ & $\mathbf{P}$ \\
\hline \multirow{4}{*}{$\begin{array}{c}\text { 'Excellent' diary } \\
\text { completion } \\
>75 \%(n=25)\end{array}$} & ASLEEP & 0.709 & 0.0001 & 0.090 & 0.6663 \\
\hline & OFF & 0.555 & 0.0040 & 0.456 & 0.0221 \\
\hline & ON & 0.704 & 0.0001 & 0.382 & 0.0596 \\
\hline & DYS & 0.690 & 0.0001 & 0.516 & 0.0083 \\
\hline \multirow{4}{*}{$\begin{array}{l}\text { 'Good' diary } \\
\text { completion } \\
>50 \%(n=29)\end{array}$} & ASLEEP & 0.430 & 0.0198 & 0.175 & 0.3637 \\
\hline & OFF & 0.470 & 0.0101 & 0.345 & 0.0668 \\
\hline & ON & 0.658 & 0.0001 & 0.262 & 0.1691 \\
\hline & DYS & 0.647 & 0.0002 & 0.516 & 0.0042 \\
\hline
\end{tabular}

\section{Figure 2}

Predictions of the ANN compared to the diary entries for three consecutive days of four participants. Each graph shows the colour-coded predictions of the ANN over the course of the day, where dark indicates high confidence, and white a lack of confidence, in each disease state over time. Solid line indicates participants' diary entries; gaps indicate missing entries. Bottom row of graphs show the daily average time spent in each disease state, according to MDS-UPDRS (UPDRS), diaries (DIA), and as predicted by ANN (ACC).

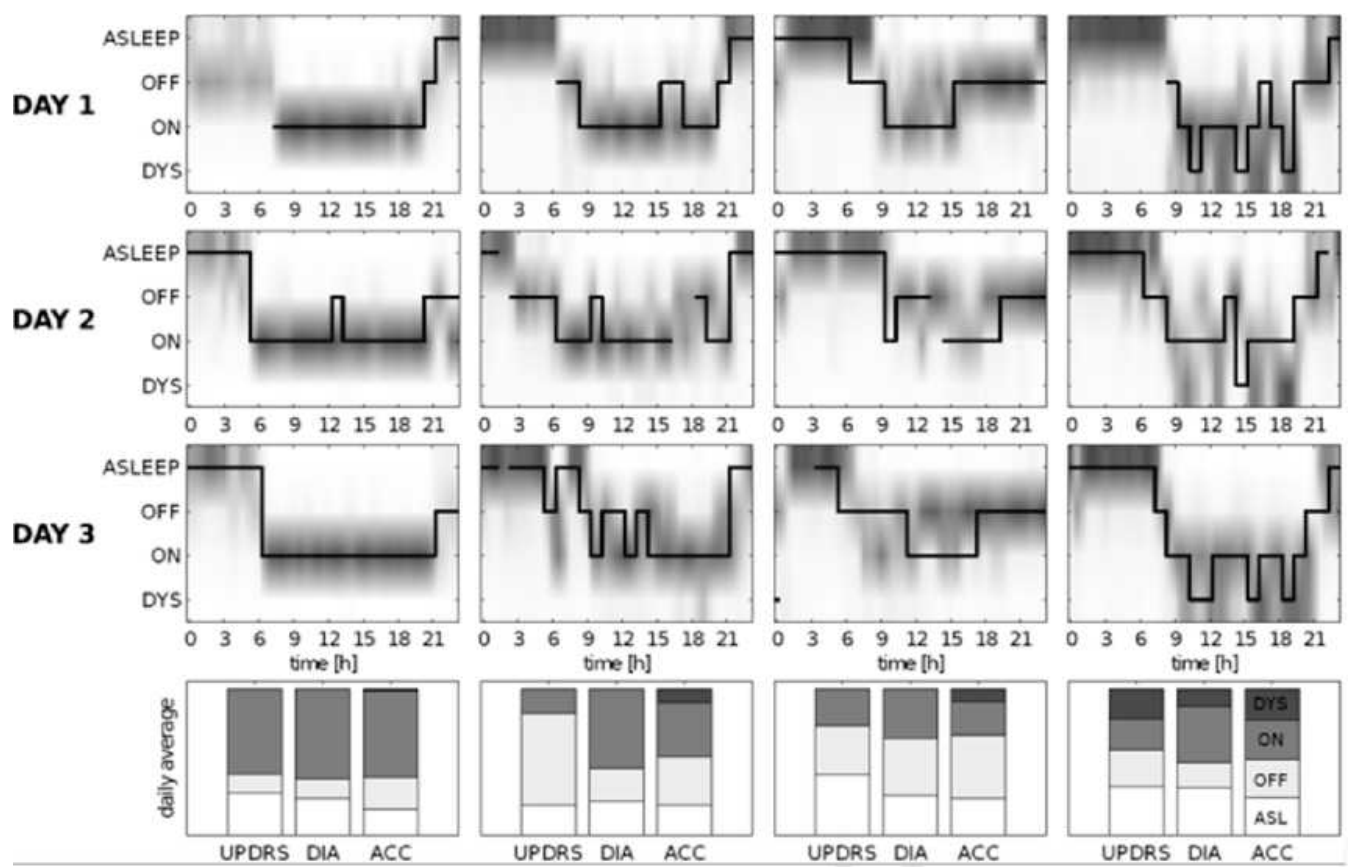

